\title{
Multi-trait BLUP in half-sib selection of annual crops
}

J. M. S. Viana ${ }^{1}$, F. M. Sobreira ${ }^{1}$, M. D. V. De Resende ${ }^{2}$ and V. R. Faria ${ }^{1}$

${ }^{1}$ Departamento de Biologia Geral, Universidade Federal de Viçosa, 36570-000, Viçosa, E-mail: jmsviana@ufv.br; ${ }^{2}$ Departamento de Engenharia Florestal, Embrapa CNPF, Universidade Federal de Viçosa, 36570-000, Viçosa, MG, Brazil

With 2 tables

Received July 28, 2009/Accepted October 28, 2009

Communicated by J. Léon

\begin{abstract}
The Best Linear Unbiased Prediction (BLUP) methodology, which is widely used in animal and forestry genetic evaluation, can also be applied to annual crop breeding. The objective of this study was to compare the accuracy and efficiency of among- and within-half-sib family selection through the use of single- and multi-trait BLUP, and BLUE/ANOVA methods. Expansion volume and yield data from two recurrent selection cycles of a popcorn population were analyzed. Progeny tests were designed as a lattice. To maximize accuracy of the prediction of breeding values, the BLUP analyses included phenotypic values of the two cycles. All BLUP analyses were performed using the ASReml software. The multi-trait BLUP method demonstrated greater accuracy and efficiency in family selection. In the case of within-family selection, both accuracy and efficiency of multi- and single-trait BLUP methods were equivalent. The selection efficiency of the multi-trait BLUP was dependent on the estimated genetic parameters, particularly the difference between the genetic and environmental correlations of the traits.
\end{abstract}

Key words: sire model — animal model — genetic evaluation

Accurate prediction of the additive genetic values of individuals under selection is desirable in any breeding programme. Henderson (1974) stated that accuracy, or the correlation between the predicted and true breeding values, is maximized through use of the Best Linear Unbiased Prediction (BLUP) with pedigree information. In breeding programmes, superior individuals are normally selected based on traits that can be correlated, due to pleiotropism, gametic phase disequilibrium, and the common influence of the environment. A bias in selection of the correlated traits can occur if those traits are analyzed individually, especially with sequential selection (Pollak et al. 1984). To avoid this type of bias, and to obtain more precise and accurate predictions of breeding values, Henderson and Quaas (1976) proposed the multi-trait BLUP method. This analysis tends to present greater accuracy because it simultaneously considers a greater amount of data and uses the genetic and residual correlations among the traits.

Multi-trait BLUP has been used in forestry and other perennial crops breeding with consistently favourable results. In a study on peach tree progenies derived from crosses involving 42 parents, De Souza et al. (1998a,b) used multi-trait BLUP to estimate genetic and phenotypic correlations. Costa e Silva et al. (2000) used single- and multi-trait BLUP in an analysis of Norway spruce (Picea abies) progenies derived from open pollination and controlled crosses. The results of their study strengthened the breeding strategy used in Denmark, based on a large central population and some small specialized populations. Multi-trait BLUP has also been used in Walnut (Juglans regia) breeding and was found to be superior to phenotypic selection (Aleta et al. 2004). In a simulation study involving two traits with different heritabilites (0.7 and 0.3), under equal, different and nil genetic and environmental correlation scenarios, and with no fixed effects and several random effects, Bauer and Léon (2008) verified greater efficiency in identifying genotypically superior pure lines and more accurate predictions through the use of multitrait BLUP. Based on their review of the use of BLUP in plant breeding, Piepho et al. (2008) stated that multi-trait BLUP is most advantageous when the traits analyzed are highly correlated. The limitations of this technique are a greater demand for computer processing, due to the slow convergence of the REML process (Piepho et al. 2008), and low parsimony, which is mainly a concern when more than three traits are considered simultaneously (Resende 2007).

The objective of this study was to compare the accuracy and efficiency of single- and multi-trait BLUP, and BLUE/ANOVA methods in annual crop breeding, using half-sib families.

\section{Materials and Methods}

Experimental data: Data from two cycles of half-sib selection in the popcorn population Viçosa were analyzed for among- and withinselection. Each cycle consisted of evaluation of 196 families in a replicated trial, selection of the best 20 progenies, recombination of these families in isolated area (recombination plot), using remnant seeds, and selection of 196 superior plants for the next cycle. The tests were designed as a $14 \times 14$ simple lattice and carried out in the experimental station of the Federal University of Viçosa (UFV) in Coimbra, Minas Gerais, Brazil, in the 1999/2000 and 2001/2002 growing seasons. The following traits were assessed in each experimental plot: final stand (covariate), yield $\left(\mathrm{kg} / 4.5 \mathrm{~m}^{2}\right)$, grain moisture $(\%$; covariate) and expansion volume $(\mathrm{EV} ; \mathrm{ml} / \mathrm{g})$, assessed in a hot air popcorn machine $(1200 \mathrm{w})$ using a $30 \mathrm{~g}$ sample. The recombination plots (areas of crosses between selected families) were conducted at the experimental station of UFV in Ponte Nova, Minas Gerais, Brazil, in the 2000/2001 and 2002/2003 growing seasons. Twenty selected families were planted, intercalating one row of male plants every four rows of female plants. Two rows were also planted with the IAC 112 single hybrid (control). Each row was $20 \mathrm{~m}$ long, with up to 100 plants. The rows of females and the control were detasseled at flowering. Twenty plants were measured in each progeny and five to 10 plants in each row of the IAC 112. Each plant was measured for yield, in $\mathrm{g} / 0.18 \mathrm{~m}^{2}$, and $\mathrm{EV}$, measured in a hot air popcorn machine using a $10 \mathrm{~g}$ sample. In the two cycles among- and within-selection were based on the Mulamba and Mock index, with weights 3 for EV and 1 for yield.

BLUP/REML analysis: The use of BLUP for among- and within-halfsib family selection aims to predict the additive genetic values of the 
common parents of the progenies and of the individuals in the recombination plot. The models for family and individual data are both commonly used in animal genetic evaluation ('sire model' and 'animal model', respectively). When there are not phenotypic values of individuals within families in the progeny test, none additional genetic variance component can be isolated from the error variance. As in any cycle with half-sib progenies the dominance genetic relationship matrix for the individuals in the recombination plot is an identity matrix, it is not possible to fit the animal model with dominance, even by combining two or more cycles. Each element in the dominance relationship matrix is the probability that the two corresponding individuals have genotypes identical by descent (Mrode 2005). Assuming the variance of the residual vector as $\boldsymbol{R}=\mathbf{I} \sigma^{2}$, as the variance of the vector of due-to-dominance genetic values equal to $\mathbf{I} \sigma_{\mathrm{D}}^{2}$, where $\sigma_{\mathrm{D}}^{2}$ is the dominance genetic variance, there is no convergence in the REML process. Then the error variance includes the dominance variance. The impossibility of adjusting the additivedominant model is common in practically all breeding experiments, including those with animal, forestry and other perennial species. In general the data permits only the adjustment of the additive model.

If $f$ families and $p$ individuals were measured for $t$ traits, then, assuming, in each cycle, assessment of the progenies in a lattice design with fixed effect of replication and random effect of blocks within replication (analysis with recovery of the interblock information), and assessment of plants in the recombination plot in the completely random design, with a genetically uniform common check (a single hybrid), the models for the analysis of the data of families and plants in the recombination plot, are given by:

$$
\begin{gathered}
\mathbf{y}=\mathbf{X} \boldsymbol{\beta}+\mathbf{Z}_{1} \mathbf{u}_{1}+\mathbf{Z}_{2} \mathbf{u}_{2}+\boldsymbol{\varepsilon} \\
\mathbf{y}=\mathbf{X} \boldsymbol{\beta}+\mathbf{Z} \mathbf{u}+\boldsymbol{\varepsilon},
\end{gathered}
$$

where $\mathbf{y}$ is the vector of phenotypic values, $\mathbf{X}$ is the incidence matrix of the fixed effects, $\beta$ is the fixed effects vector, $\mathbf{Z}_{1}, \mathbf{Z}_{2}$ and $\mathbf{Z}$ are the incidence matrices of the random effects, $\mathbf{u}_{1}$ is the vector of block|replication/generation effects, $\mathbf{u}_{2}$ is the vector of the additive genetic values of the common parents of the families divided by 2 , $\mathbf{u}$ is the vector of the additive genetic values of the individuals in the recombination plot, and $\varepsilon$ is the residual vector, all relative to the $t$ traits. Both models include a fixed effect of generation (confounded with the effect of year). In relation to the analysis of individual data, the model includes a fixed effect of population and a fixed effect of interaction population $\times$ generation. The ASReml programs are presented in the Appendix.

The criterion used to obtain the best linear unbiased predictor of a random vector is the maximization of the joint probability density function of $\mathbf{y}$ and the random vector(s), which obtains, under normality, the mixed model equations (MME) (Henderson 1974). Alternatively the predictors can be obtained by maximizing the joint probability density function of the random vector(s) and $\varepsilon$. Regarding the model $\mathbf{y}=\mathbf{X} \boldsymbol{\beta}+\mathbf{Z}_{1} \mathbf{u}_{1}+\mathbf{Z}_{2} \mathbf{u}_{2}+\boldsymbol{\varepsilon}$ the MME are:

$$
\left[\begin{array}{ccc}
\mathbf{X}^{\prime} \mathbf{R}^{-1} \mathbf{X} & \mathbf{X}^{\prime} \mathbf{R}^{-1} \mathbf{Z}_{1} & \mathbf{X}^{\prime} \mathbf{R}^{-1} \mathbf{Z}_{2} \\
\mathbf{Z}_{1}^{\prime} \mathbf{R}^{-1} \mathbf{X} & \mathbf{Z}_{1}^{\prime} \mathbf{R}^{-1} \mathbf{Z}_{1}+\mathbf{G}_{1}^{-1} & \mathbf{Z}_{1}^{\prime} \mathbf{R}^{-1} \mathbf{Z}_{2} \\
\mathbf{Z}_{2}^{\prime} \mathbf{R}^{-1} \mathbf{X} & \mathbf{Z}_{2}^{\prime} \mathbf{R}^{-1} \mathbf{Z}_{1} & \mathbf{Z}_{2}^{\prime} \mathbf{R}^{-1} \mathbf{Z}_{2}+\mathbf{G}_{2}^{-1}
\end{array}\right]\left[\begin{array}{c}
\boldsymbol{\beta}^{o} \\
\tilde{\mathbf{u}}_{1} \\
\tilde{\mathbf{u}}_{2}
\end{array}\right]=\left[\begin{array}{c}
\mathbf{X}^{\prime} \mathbf{R}^{-1} \mathbf{y} \\
\mathbf{Z}_{1}^{\prime} \mathbf{R}^{-1} \mathbf{y} \\
\mathbf{Z}_{2}^{\prime} \mathbf{R}^{-1} \mathbf{y}
\end{array}\right]
$$

In the single-trait analysis, $\mathbf{R}=\sigma^{2} \mathbf{I}_{(n)}$ ( $n$ is the number of observations); $\mathbf{G}_{1}=\sigma_{b}^{2} \mathbf{I}_{(b)}$ ( $b$ is the number of blocks); and $\mathbf{G}_{2}=(1 / 4) \sigma_{\mathrm{A}}^{2} \mathbf{A}_{(f)}$, where $\mathbf{A}_{(f)}$ is the additive genetic relationship matrix of the common parents of the families.

In the multi-trait analysis, $\mathbf{R}=\operatorname{Cov}(\mathbf{E})_{(t)} \otimes \mathbf{I}_{(n)}$, and $\operatorname{Cov}(E)_{(t)}$ is the residual variance matrix relative to the $\mathrm{t}$ traits; $\mathbf{G}_{1}=$ $\operatorname{Cov}(\mathbf{B}|\mathbf{R}| \mathbf{G})_{(t)} \otimes \mathbf{I}_{(b q)}$, and $\operatorname{Cov}(\mathrm{B}|\mathrm{R}| \mathrm{G})_{(t)}$ is the variance matrix of the block|replication/generation effects, relative to the $t$ traits $(g$ is the number of generations); and $\mathbf{G}_{2}=(1 / 4) \cdot \operatorname{Cov}(\mathbf{A})_{(t)} \otimes \mathbf{A}_{(f)}$, and $\operatorname{Cov}(\mathbf{A})_{(t)}$ is the additive genetic variance matrix, relative to the $t$ traits.

Regarding the model $\mathbf{y}=\mathbf{X} \boldsymbol{\beta}+\mathrm{Zu}+\boldsymbol{\varepsilon}, \operatorname{Var}(\mathrm{u})=\mathbf{G}=\sigma_{\mathrm{A}}^{2} \mathbf{A}_{(p)}$, in the single-trait analysis, and $\operatorname{Var}(\mathrm{u})=\mathbf{G}=\operatorname{Cov}(\mathbf{A})_{(t)} \otimes \mathbf{A}_{(p)}$, in the multi- trait analysis, where $\mathbf{A}_{(p)}$ is the additive genetic relationship matrix of the individuals in the recombination plot.

The accuracies of the single- and multi-trait analyses are given by: $\mathrm{r}_{\mathrm{A} \tilde{\mathrm{A}}}=\sqrt{1-\left(\frac{\mathrm{PEV}}{(1 / 4) \tilde{\sigma}_{\mathrm{A}}^{2}}\right)}$, relative to the breeding values predicted by the sire model, $\mathrm{r}_{\mathrm{A} \tilde{A}}=\sqrt{1-\left(\frac{\mathrm{PEV}}{\tilde{\sigma}_{\mathrm{A}}^{2}}\right)}$, relative to breeding values predicted by the animal model, and where PEV is the prediction error variance and $\tilde{\sigma}_{\mathrm{A}}^{2}$ is the restricted maximum likelihood estimator of the additive variance (Mrode 2005)

Assuming population in Hardy-Weinberg equilibrium the mean of the population obtained by mass selection on only one gender can be expressed as (Viana et al. 2009):

$$
M_{1}=M+(1 / 2) E\left(A_{\mathrm{s}}\right),
$$

where $M$ is the mean of the base population and $E\left(A_{\mathrm{s}}\right)$ is the expectation of the additive genetic values of the selected individuals. In the case of among half-sib selection, with recombination of all progenies, the mean of the improved population can be expressed as (Viana et al. 2009):

$$
M_{1}=M+(1 / 2) E\left(\frac{1}{2} A_{\mathrm{s}}\right)
$$

Assuming, as Eberhart (1970), that the expected genetic gain with selection on both genders is twice the expected gain with selection on only one gender, the predicted genetic gain can be computed from parental control $(c)$ and the predicted breeding values of the selected individuals $\left(\tilde{A}_{\mathrm{S}}\right)$ by:

$$
\Delta M_{\mathrm{e}}=c\left(\frac{1}{n_{\mathrm{s}}} \sum_{i} k \tilde{A}_{\mathrm{S}_{\mathrm{i}}}\right),
$$

where $n_{\mathrm{s}}$ is the number of selected families or plants (20 and 196, for among- and within-selection), and $c=1$ and $k=1 / 2$ for family selection, or $c=1 / 2$ and $k=1$ for selection in the recombination plot.

This function is also obtained by an equivalent derivation of that used by Eberhart (1970), assuming that the predicted gain is proportional to a selection differential based on predicted breeding values $\left(\Delta M_{\mathrm{e}}=b\left(\bar{A}_{\mathrm{S}}-\bar{A}\right)\right.$, where $\bar{A}_{\mathrm{S}}$ is the mean of the breeding values of the selected individuals and $\bar{A}$ is the mean of the predicted breeding values). Considering mass selection on only one gender and that the coefficient of proportionality is the regression coefficient of the genotypic value of half-sib family in the improved population $\left(\bar{G}_{\mathrm{o}}\right)$ as a function of the additive genetic value of its common parent (selected individual), we have:

$$
b=\frac{\operatorname{Cov}\left(A_{\mathrm{s}}, \bar{G}_{\mathrm{o}}\right)}{V\left(A_{\mathrm{s}}\right)}
$$

Assuming irrelevant changes in gene frequencies, $b=1 / 2$. In general, $b=2 r_{X Y}$, where $r$ is the coefficient of relationship, $X$ is selected individual and $Y$ is it descendent in the improved population. With half-sib selection on only one gender, $b=1 / 4$.

The derived function of predicting response to selection is more biased with selection on both genders, since with mass selection before flowering and half-sib selection with recombination only of selected progenies the mean of the improved population are, respectively:

$$
\begin{gathered}
M_{1}=M+(1 / 2) E\left(A_{\mathrm{s}}\right)+E(f) \\
M_{1}=M+(1 / 2) E\left(\frac{1}{2} A_{\mathrm{s}}\right)+E\left(f^{\prime}\right)
\end{gathered}
$$

where the expectations of $f$ and $f^{\prime}$ are functions of $\Delta p$ (change in the favourable gene frequency), $a$ [difference between the genotypic value of the homozygote of greater expression and the mean of the genotypic values of the homozygotes $(m)$ ], $d$ (difference between the genotypic value of the heterozygote and $m$ ), and $p$ (frequency of favourable gene) [only $\mathrm{E}\left(f^{\prime}\right)$ ], for each gene. For a gene the parametric values of $f$ and $f^{\prime}$ are: 
$f=\Delta p(a-d) \quad$ or $\quad \Delta p(a)$ or $\quad \Delta p(a+d)$

$f^{\prime}=\Delta p(a-p d) \quad$ or $\quad \Delta p[a+0.5(1-2 p) d] \quad$ or $\quad \Delta p[a+(1-p) d]$

if the common parent is homozygous for the gene that increase trait expression, heterozygous or homozygous for the gene that decrease trait expression, respectively.

The function of response to selection based on predicted breeding values was used to predict direct and indirect gains with selection on EV and, with multi-trait BLUP, with selection based on the rank summation index (Mulamba and Mock 1978). The weights of predicted breeding values for EV and yield were 3 and 1 .

Other functions of response to selection were proposed by Cullis et al. (2006) and Piepho and Möhring (2007). In the former paper the predicted gain is calculated from a generalized measure of heritability, obtained from the average pairwise prediction error variance (average of $\left.\operatorname{Var}\left(\tilde{u}_{i}-\tilde{u}_{j}\right)=\operatorname{Var}\left(\tilde{u}_{i}\right)+\operatorname{Var}\left(\tilde{u}_{j}\right)-2 \cdot \operatorname{Cov}\left(\tilde{u}_{i}, \tilde{u}_{j}\right)\right)$. This requires the estimated prediction error variance matrix. An alternative generalized measure of heritability was proposed by Oakey et al. (2006). Its computation requires multiply the inverse of the additive relationship matrix and the estimated prediction error variance matrix. The predicted gain proposed by Piepho and Möhring (2007) is the average value of the response to selection proposed in this paper, obtained from a large number of simulations, each one derived from the original data by bootstrap. For comparison purpose the genetic gains were also computed from the function proposed by Cullis et al. (2006), including the parental control (c). The indirect expected genetic gain in relation to trait $Y$ with selection based on trait $X$ is:

$$
\Delta M_{\mathrm{e} Y(X)}=\text { c.i. } \rho_{\mathrm{g}_{X, Y}} \sigma_{g_{Y}} h_{X},
$$

where $i$ is the selection intensity, $\rho_{\mathrm{g}_{X, Y}}$ is the genetic correlation, $\sigma_{\mathrm{g}_{Y}}^{2}$ is the genotypic variance and $h_{X}^{2}$ is the generalized measure of heritability.

The ASReml software (Gilmour et al. 2006) was used for all BLUP analyses. As the model for bi-trait analysis of family data cannot include covariates for yield (but not for expansion volume), the multigeneration analyses were made with yield corrected for stand and grain moisture using regression coefficients estimates from initial BLUP single-generation analyses with covariates.

BLUE/ANOva analysis: An additional analysis, normally employed by breeders, was made only with data from the second cycle. This is characterized by no inclusion of pedigree information, estimation of the variance components by the ANOVA method (Henderson's type III method), selection based on adjusted means or phenotypic values, and prediction of genetic gain by the function proposed by Eberhart (1970):

$$
\Delta M_{\mathrm{e}}=c \cdot \mathrm{SD} \cdot h^{2}
$$

where $c$ is the parental control, SD is the selection differential and $h^{2}$ is the heritability, calculated as $(1 / 4) \sigma_{\mathrm{A}}^{2} / \sigma_{\mathrm{Pf}}^{2}$ and $\sigma_{\mathrm{A}}^{2} / \sigma_{\mathrm{P}}^{2}$ for progenies and plants, where $\sigma_{\mathrm{P}}^{2}$ stands for phenotypic variance.

For this analysis we employed the MIXED procedure of the SAS System. The accuracy of this analysis is the square root of the heritability. For family data the accuracy is the correlation between the family phenotypic mean and the additive genetic value of the common parent. For individual data the accuracy is the correlation between the phenotypic and breeding values.

\section{Results}

\section{Among family selection (sire model)}

The estimates of the additive (four times the genotypic variance) and residual variances were very close between the multi-trait and single-trait BLUP analyses (Table 1). The multi-trait BLUP gave superior accuracy compared with the single-trait analysis and BLUE/AnOva. Compared with the single-trait BLUP, the multi-trait analysis presented accuracy values 5.9 and $4.8 \%$ superior for EV and yield, respectively. The accuracy of the multi-trait and single-trait analyses were 38.4 and $30.7 \%$ superior to BLUE/ANOVA for EV and 4.7 and $0.0 \%$ superior for production, respectively. The genetic correlation between EV and yield was negative $(-0.39)$ and the residual correlation was reduced $(-0.01)$. Based on predicted gains in EV, since quality is more important than yield, selection based on the breeding values predicted by the multitrait analysis was more efficient, with increases of 5 and $42 \%$ compared with single-trait analysis and BLUE/ANOVA, respectively (Table 1). The expected gains based on the generalized measure of heritability were of inferior magnitude in relation to expected gains based on the predicted breeding values (73$93 \%$ ), but concordant in sign. In the multi-trait BLUP analysis the use of the proposed index gives the better genetic gains: the gain in $\mathrm{EV}$ is $97 \%$ of the maximum value and the negative response in yield is $50 \%$ less.

Regarding the coincidence of the 20 families selected in the second cycle by the multi-trait and single-trait predictions of the breeding values and by phenotypic means based on the estimated family mean, differences were observed among the methods. Seventy-five and $80 \%$ of the families selected based on EV and on yield by the multi-trait analysis were also selected by the single-trait analysis. Based on phenotypic means, however, only $60 \%$ of the families selected based on

\begin{tabular}{|c|c|c|c|c|c|c|}
\hline \multirow[b]{2}{*}{ Parameters } & \multicolumn{2}{|c|}{ Multi-trait BLUP ( 2 cycles) } & \multicolumn{2}{|c|}{ Single-trait BLUP ( 2 cycles $)$} & \multicolumn{2}{|c|}{ BLUE/ANOVA (cycle 2) } \\
\hline & $\mathrm{EV}(\mathrm{ml} / \mathrm{g})$ & Yield (kg/ha) & $\mathrm{EV}(\mathrm{ml} / \mathrm{g})$ & Yield (kg/ha) & $\mathrm{EV}(\mathrm{ml} / \mathrm{g})$ & Yield $(\mathrm{kg} / \mathrm{ha})$ \\
\hline Genotypic variance & $1.8702^{0.0025}$ & $102179.0^{0.0017}$ & $1.7523^{0.0043}$ & $98129.8^{0.0025}$ & $1.4566^{0.0201}$ & $47463.9^{0.0058}$ \\
\hline Block $\mid$ Rep $\mid$ Gen. var. & 1.8779 & 41502.2 & 1.8672 & 43443.1 & 1.6303 & 82995.0 \\
\hline Residual variance & 9.9120 & 539872.0 & 10.0063 & 541933.0 & 7.3765 & 189063.6 \\
\hline Heritability ${ }^{1,2}$ & 0.3244 & 0.3294 & 0.2875 & 0.2977 & 0.2644 & 0.3161 \\
\hline Accuracy & 0.5786 & 0.5864 & 0.5461 & 0.5594 & 0.4179 & 0.5602 \\
\hline Direct gain & 1.48 & & 1.41 & & 1.04 & \\
\hline Indirect gain & & -172.86 & & 3.39 & & -13.43 \\
\hline Direct gain $^{2}$ & 1.37 & & 1.25 & & & \\
\hline Indirect gain $^{2}$ & & -125.71 & & - & & \\
\hline Gain w/sel. index ${ }^{4}$ & 1.43 & -86.99 & & & & \\
\hline Genetic correlation & & 934 & & & & \\
\hline Residual correlation & & 093 & & & & \\
\hline Phenotypic correlation & & 541 & & & & \\
\hline
\end{tabular}

Table 1: Estimates of genetic and non-genetic parameters from BLUP/REML and BLUE/ANOva (fixed model) using family data

${ }^{1}$ Generalized measure of heritability for BLUP analysis; ${ }^{2}$ from Cullis et al. (2006); ${ }^{3}$ absolute value $>1 ;{ }^{4}$ Mulamba and Mock (1978). 
EV and $45 \%$ of those selected based on yield were also selected by the multi-trait BLUP.

\section{Within-family selection (animal model)}

The estimates of the additive and residual variances were also very similar between the multi-trait and single-trait BLUP analyses (Table 2). The accuracy and selection efficiency of the multi- and single-trait BLUP analyses were equivalent. Compared with BLUE/ANOVA, however, the accuracy values of the two BLUP methods were inferior for EV. In the BLUP analyses the genetic correlations were reduced $(0.02$ and 0.03 approximately) and the residual ( 0.11 and 0.10 approximately) correlations were superior to the genetic correlations. The predicted gains in EV by single- and multi-trait BLUP analyses were approximately equal (Table 2), and about $15 \%$ superior to the gain obtained with BLUE/ANOVA. The indirect gains for yield were irrelevant in the BLUP analyses. It is interesting to note that in the case of within-family selection the expected gains based on the generalized measure of heritability were, in general, 52-140\% superior to the expected gains based on the predicted breeding values. With multi-trait BLUP analysis, the best predicted gains were also obtained with selection based index. The value predicted for $\mathrm{EV}$ is $98 \%$ of the maximum value, associated with positive gain in yield.

In relation to the coincidence involving the 196 individuals selected in the second cycle recombination plot, 98 and $99.5 \%$ of the individuals selected by the multi-trait analysis based on EV or on yield respectively, were also selected by the singletrait analysis. The multi-trait BLUP and the BLUE/ANOvA were also equivalent. The coincidences of selected individuals were 98 and $99 \%$ for EV and yield.

\section{Discussion \\ Among-family selection (sire model)}

The greater accuracy and selection efficiency of the multi-trait BLUP method, as compared with the single-trait analysis, was mainly due to the difference between the genetic and the environmental correlations relative to yield and EV (Table 1). According to Schaeffer (1984) and Thompson and Meyer (1986), the increase in accuracy obtained by using multi-trait BLUP is proportional to the absolute difference between the genetic and environmental correlations of the traits. When the heritability of the traits are equal, the selection efficiency of multi-trait BLUP, compared with single-trait analysis, depends only on the absolute difference between the genetic and environmental correlations of the variables analyzed (Resende 2007).

Our results corroborate those obtained by Bauer and Léon (2008) and by Persson and Andersson (2004). In the second study, the authors used Monte Carlo simulation to compare the prediction of the breeding values via multi-trait and singletrait BLUP, considering different values of genetic and environmental correlations and heritabilities. The authors observed that the multi-trait predictors resulted in a smaller average bias, and were therefore considered to be more accurate. Greater accuracy and efficiency of the multi-trait BLUP were also reported by Da Costa et al. (2002) in rubber tree breeding (Hevea brasiliensis), as well as by Kerr (1998) in the selection of open and controlled cross pollination cultivars in forestry breeding. These authors reported that an increase in the number of traits considered as selection criteria gave a greater advantage to the multi-trait analysis. When three traits were used as selection criteria, the increase in accuracy of the multi-trait analysis was less than $1 \%$ for all cultivars. However, when five traits were used as selection criteria, the increase in accuracy of the multi-trait analysis was 3\% for the open pollination cultivars and $1.3 \%$ for the controlled pollination cultivars. Kadarmideen et al. (2003) compared the prediction accuracy of the breeding values for fertility by single-trait BLUP with the accuracy of the multi-trait analysis with fertility and milk production traits in dairy cattle. Both the predicted breeding values and the ranking of the animals were significantly different between the two analyses. The multi-trait analysis improved the accuracy of the predicted breeding values for each trait involved by reducing the prediction error variance. Multi-trait BLUP analysis of fertility and milk production was recommended by the authors, who argued that this analysis is efficient for traits that present unfavourable genetic correlation. Another advantage of the multi-trait BLUP observed in this study is the fact that this analysis permits prediction of the breeding values of individuals for a trait that was not assessed (fertility) through the assessment of a correlated trait (milk production). This extension allowed for prediction of the breeding values for fertility to be obtained for animals assessed only for milk production.

Compared with single-trait BLUP based only on the second cycle family test (not published), the multi-trait analysis

Table 2: Estimates of genetic and non-genetic parameters from BLUP/REML and BLUE/ANOva (fixed model) using individual data

\begin{tabular}{|c|c|c|c|c|c|c|}
\hline \multirow[b]{2}{*}{ Parameters } & \multicolumn{2}{|c|}{ Multi-trait BLUP (2 cycles) } & \multicolumn{2}{|c|}{ Single-trait BLUP (2 cycles) } & \multicolumn{2}{|c|}{ BLUE/ANOVA (cycle 2) } \\
\hline & $\mathrm{EV}(\mathrm{ml} / \mathrm{g})$ & Yield $\left(\mathrm{g} / 0.18 \mathrm{~m}^{2}\right)$ & $\mathrm{EV}(\mathrm{ml} / \mathrm{g})$ & Yield $\left(\mathrm{g} / 0.18 \mathrm{~m}^{2}\right)$ & $\mathrm{EV}(\mathrm{ml} / \mathrm{g})$ & Yield $\left(\mathrm{g} / 0.18 \mathrm{~m}^{2}\right)$ \\
\hline Additive variance & $16.8424^{8.4 \mathrm{E}-8}$ & $221.5740^{0.0017}$ & $16.5041^{1.5 \mathrm{E}-7}$ & $220.7670^{0.0016}$ & 18.4719 & 519.4500 \\
\hline Residual variance & 11.1404 & 619.276 & 11.3845 & 619.912 & 8.1000 & 282.7100 \\
\hline Heritability ${ }^{1,2}$ & 0.5301 & 0.2989 & 0.5221 & 0.2979 & 0.6952 & 0.6476 \\
\hline Accuracy & 0.7955 & 0.8755 & 0.7892 & 0.8758 & 0.8327 & 0.8037 \\
\hline Direct gain & 1.71 & & 1.69 & & 1.48 & \\
\hline Indirect gain & & -0.17 & & 0.10 & & 1.72 \\
\hline Direct gain $^{2}$ & 2.62 & & 2.57 & & & \\
\hline Indirect gain ${ }^{2}$ & & 0.15 & & 0.24 & & \\
\hline Gain w/sel. Index ${ }^{3}$ & 1.67 & 0.84 & & & & \\
\hline Genetic correlation & \multicolumn{2}{|c|}{0.0162} & \multicolumn{2}{|c|}{0.0262} & \multicolumn{2}{|r|}{-0.1500} \\
\hline Residual correlation & \multicolumn{2}{|c|}{0.1080} & \multicolumn{2}{|c|}{0.1008} & \multicolumn{2}{|r|}{0.7000} \\
\hline Phenotypic correlation & \multicolumn{2}{|c|}{0.0121} & \multicolumn{2}{|c|}{0.0121} & \multicolumn{2}{|r|}{0.1296} \\
\hline
\end{tabular}

${ }^{1}$ Generalized measure of heritability for BLUP analysis; ${ }^{2}$ from Cullis et al. (2006); ${ }^{3}$ Mulamba and Mock (1978). 
considering the first two cycles gave more accurate breeding values for yield (0.87 vs. 0.37$)$, greater predicted gain in EV $(1.48 \mathrm{ml} / \mathrm{g}$ vs. $0.40 \mathrm{ml} / \mathrm{g})$ and greater negative indirect gains in yield $(-172.86$ vs. $-12.42 \mathrm{~kg} / \mathrm{ha})$.

Under lower selection intensity (40 selected families), the coincidences among the families selected by the different analyses were greater for EV $(90 \%)$ and yield $(82.5 \%)$, for the single-trait and multi-trait BLUP. In relation to BLUE/ANOVA, $72.5 \%$ of the families selected for EV and $57.5 \%$ of the families selected for yield would also be selected by the multi-trait analysis.

\section{Within-family selection (animal model)}

The multi-trait BLUP demonstrated accuracy equivalent to the single-trait analysis, showing that under the conditions studied, multi-trait analysis was not advantageous (Table 2). As emphasized, the decision to use multi-trait BLUP rather than a single-trait analysis would depend mainly on the heritabilities and correlations of the traits. Depending on the combination of these parameters, the efficiency of these two models may be similar. Thus, in situations where all the traits are observed for each plant, the heritabilities of the traits are similar, and all traits are favourably correlated, analysis with a model that considers multiple traits may not provide a substantial increase in the accuracy of the predicted breeding values (Schaeffer 1984). As the difference between the genetic and environmental correlations for EV and yield was reduced, no advantage was demonstrated by using multi-trait BLUP. Jain and Sadana (2000) compared accuracy and selection efficiency in bulls, using conventional, single-trait and multi-trait BLUP methods, following the animal model. Their results showed that multi-trait analysis can be either more or less accurate than the single-trait analysis, depending on the genetic parameters estimated.

For within family selection, multi-trait analysis combining the first two cycles surpassed the single-trait analysis using data from only the second cycle (not published), relative to EV predicted gain $(1.71 \mathrm{ml} / \mathrm{g}$ vs. $0.98 \mathrm{ml} / \mathrm{g})$. The estimated indirect gains in yield were different in magnitude and sign $(-0.17 \mathrm{~g} /$ $0.18 \mathrm{~m}^{2}$ vs. $0.85 \mathrm{~g} / 0.18 \mathrm{~m}^{2}$ ). The accuracy of prediction was inferior with multi-trait BLUP, for both traits.

\section{Acknowledgements}

We thank the Foundation for Research Support of Minas Gerais State (Fapemig), the Brazilian Federal Agency for Support and Evaluation of Graduate Education (Capes) and the National Council for Scientific and Technological Development (CNPq) for financial support.

\section{Appendix}

\section{ASReml code for the single-trait BLUP analysis}

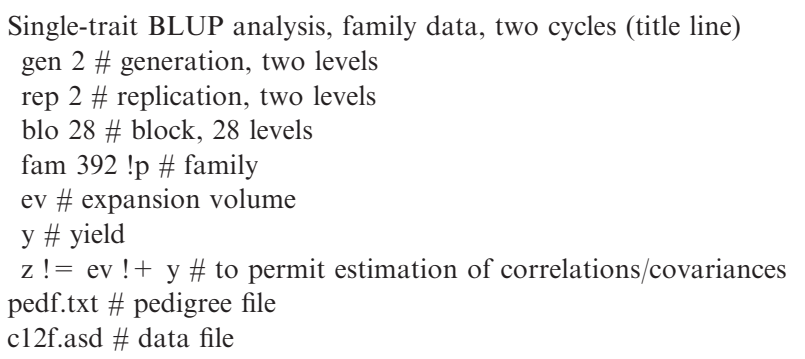

!ddf \# correction of df by the Kenward-Roger method ev or y or $\mathrm{z} \sim$ mu gen gen.rep !r gen.rep.blo fam predict fam !vpv \# to save file .pvs with the prediction error variance matrix

Single-trait BLUP analysis, individual data, two cycles gen 2

pop 2 \# population, two levels

ind 801 !p \# individual, IAC 112 coded as 801

ev

$\mathrm{y}$

$\mathrm{z} !=\mathrm{ev} !+\mathrm{y}$

pedp.txt \# pedigree file

c12p.asd \# data file

!ddf

ev or y or $\mathrm{z} \sim \mathrm{mu}$ gen pop pop.gen !r ind

predict ind !vpv

\section{ASReml code for the multi-trait BLUP analysis}

Multi-trait BLUP analysis, family data, two cycles

gen 2

rep 2

blo 28

fam 392 !p

ev

y

pedf.txt

c12f.asd

!ddf

ev y $\sim$ Trait Trait.gen Trait.gen.rep !r Trait.gen.rep.blo Trait.fam

122 \# one R structure (product of two matrices) and two G structures 00 ID

Trait 0 US 101000550000 \# var(ev) cov(ev, y) var(y); initial values

Trait.gen.rep.blo 2 \# G1 structure as a product of two matrices

Trait 0 CORR 0245000 \# corr(ev, y) var(ev) var(y); initial values gen.rep.blo 0 ID

Trait.fam 2 \# G2 structure as a product of two matrices

Trait 0 CORR 02100000 \# initial values

fam

Predict fam Trait !vpv

Multi-trait BLUP analysis, individual data, two cycles

gen 2

pop 2

ind $801 ! p$

ev

y

pedp.txt

c12p.asd

!ddf

ev y $\sim$ Trait Trait.gen Trait.pop Trait.gen.pop !r Trait.ind

121 \# one G structure

00 ID

Trait 0 US 1510600

Trait.ind 2

Trait 0 CORR 020250

ind

Predict ind Trait !vpv

\section{References}

Aleta, N., A. Ninot, and J. Voltas, 2004: Retrospective evaluation of parental selection in nursery tests of Juglans regia L. using a mixed model analysis. Silvae Genet. 53, 26-33.

Bauer, A. M., and J. Léon, 2008: Multiple-trait breeding values for parental selection in self-pollinating crops. Theor. Appl. Genet. 116, $235-242$. 
Costa e Silva, J., H. Wellendorf, and N. M. G. Borralho, 2000: Prediction of breeding values and expected genetic gains in diameter growth, wood density and spiral grain from parental selection in Picea abies (L.) KARST. Silvae Genet. 49, 102-109.

Cullis, B. R., A. B. Smith, and N. E. Coombes, 2006: On the design of early generation variety trials with correlated data. J. Agric. Biol. Environ. Stat. 11, 381-393.

Da Costa, R. B., M. D. V. Resende, P. S. Gonçalves, and M. A. Silva, 2002: Individual multivariate REML/BLUP in the presence of genotype $\mathrm{x}$ environment interaction in rubber tree breeding. Crop Breed. Appl. Biotechnol. 2, 131-140.

De Souza, V. A. B., D. H. Byrne, and J. F. Taylor, 1998a: Heritability, genetic and phenotypic correlations, and predicted selection response of quantitative traits in peach: I. An analysis of several reproductive traits. J. Am. Soc. Hortic. Sci. 123, $598-603$.

De Souza, V. A. B., D. H. Byrne, and J. F. Taylor, 1998b: Heritability, genetic and phenotypic correlations, and predicted selection response of quantitative traits in peach: II. An analysis of several fruit traits. J. Am. Soc. Hortic. Sci. 123, 604-611.

Eberhart, S. A., 1970: Factors effecting efficiencies of breeding methods. African Soils 15, 669-680.

Gilmour, A. R., B. J. Gogel, B. R. Cullis, and R. Thompson, 2006 ASReml User Guide Release 2.0. VSN International Ltd, Hemel Hempstead, HP1 1ES, UK.

Henderson, C. R., 1974: General flexibility of linear model techniques for sire evaluation. J. Dairy Sci. 57, 963-972.

Henderson, C. R., and R. L. Quaas, 1976: Multiple trait evaluation using relatives records. J. Anim. Sci. 43, 1188-1197.

Jain, A., and D. K. Sadana, 2000: Sire evaluation using animal model and conventional methods in Murrah buffaloes. Asian-australas. J. Anim. Sci. 13, 1196-1200.

Kadarmideen, H. N., R. Thompson, M. P. Coffeyc, and M. A. Kossaibati, 2003: Genetic parameters and evaluations from single and multiple trait analysis of dairy cow fertility and milk production. Livest. Prod. Sci. 81, 183-195.

Kerr, R. J., 1998: Asymptotic rates of response from forest tree breeding strategies using best linear unbiased prediction. Theor. Appl. Genet. 96, 484- 493.

Mrode, R. A., 2005: Linear Models for the Prediction of Animal Breeding Values, 2nd edn. CABI Publishing, Oxfordshire.

Mulamba, N. N., and J. J. Mock, 1978: Improvement of yield potential of Eto Blanco maize (Zea mays L.) population by breeding for plant traits. Egypt. J. Genet. Cytol. 1, 40-57.

Oakey, H., A. Verbyla, W. Pitchford, B. Cullis, and H. Kuchel, 2006: Joint modeling of additive and non-additive genetic line effects in single field trials. Theor. Appl. Genet. 113, 809-819.

Persson, T., and B. Andersson, 2004: Accuracy of single and multipletrait REML evaluation of data including non-random missing records. Silvae Genet. 53, 135-139.

Piepho, H. P., and J. Möhring, 2007: Computing heritability and selection response from unbalanced plant breeding trials. Genetics 177, 1881-1888.

Piepho, H. P., J. Möhring, A. E. Melchinger, and A. Büchse, 2008: BLUP for phenotypic selection in plant breeding and variety testing. Euphytica 161, 209-228.

Pollak, E. J., J. Van der Werf, and R. L. Quaas, 1984: Selection bias and multiple trait evaluation. J. Dairy Sci. 67, 1590-1595.

Resende, M. D. V., 2007: Matemática e Estatística na Análise de Experimentos e no Melhoramento Genético. Embrapa, Colombo.

Schaeffer, L. R., 1984: Sire and cow evaluation under multiple traits model. J. Dairy Sci. 67, 1567-1580.

Thompson, R., and K. Meyer, 1986: A review of theoretical aspects in the estimation of breeding values for multi-trait selection. Livest. Prod. Sci. 15, 299-313.

Viana, J. M. S., V. R. Faria, and A. C. Silva, 2009: Bias in the prediction of genetic gain due to mass and half-sib selection in random mating populations. Genet. Mol. Biol. 32, 497-506. 